\title{
Mínimos quadrados lineares desenvolvidos no contexto do Ensino Médio
}

\author{
Adriano R. de Melo
}

\begin{abstract}
Resumo
Estudamos o método dos mínimos quadrados lineares na perspectiva de conteúdos do ensino médio, a saber, funções de segundo grau e desigualdades, compreendendo-o como um problema de otimização que pode ser acessível ao ensino médio, sobretudo quando desenvolvido na forma de projetos de ensino e iniciação científica em classes de cursos técnicos de tecnologia integrados ao ensino médio.
\end{abstract}

Palavras-chave: Função de Segundo Grau; Otimização no Ensino Médio; Aplicações no Ensino Médio.

\section{Abstract}

We study the method of linear least squares in the perspective of high school contents, namely, quadratic functions and inequalities, understanding it as an optimization problem that can be accessible to high school, especially when developed in the form of teaching projects and scientific initiation in classes of technology courses integrated to high school.

Keywords: Quadratic Function; Optimization in High School; Applications in High School.

\section{Introdução}

O primeiro contato com problemas de otimização ocorre, normalmente, no ensino médio ao se estudar as funções quadráticas, que se constituem como o primeiro tipo de função ao qual se é possível associar os termos máximo e mínimo (no contexto de domínio real), facilmente intuídos como a ordenada do vértice da parábola correspondente. Embora a análise gráfica seja sensível, concreta e lúdica, pode-se definir esse conceito formalmente como:

Definição 1. Uma função $f$, com domínio em $\mathbb{R}$, possui um mínimo global quando se é possível encontrar $x_{m} \in \mathbb{R}$, tal que $f\left(x_{m}\right) \leq f(x)$, qualquer que seja $x \in \mathbb{R}$. Analogamente, o máximo global é definido a partir da possibilidade de se poder determinar $x_{M} \in \mathbb{R}$ de tal modo que $f\left(x_{M}\right) \geq f(x)$, para todo $x \in \mathbb{R}$.

Nessa fase de escolarização, os principais e convencionais exemplos que tratam deste tópico trazem por objetivo a minimização ou maximização de áreas, lucros, receitas, alturas ou velocidades de projéteis (IEZZI et al., 2013; PAIVA, 2015; CHAVANTE, PRESTES, 2016; SOUZA, GARCIA, 2016). 
Diante dessa lista de temas, perguntamo-nos se não existiria um problema de otimização de função quadrática mais sofisticado, porém acessível ao aluno do ensino médio. Pensamos no clássico da teoria da aproximação: a técnica de mínimos quadrados lineares, comumente estudada no nível de graduação nas disciplinas de estatística (como uma técnica da análise de regressão linear) e cálculo numérico. Ora, seriam os argumentos lógicos de sua construção compatíveis com o nível médio de ensino? Este artigo propõe responder positivamente essa questão.

\subsection{O Contexto do Ensino Médio}

No contexto do ensino médio, um problema clássico e de simples solução (IEZZI, MURAKAMI, $1977)$ é aquele em que se deseja determinar, para dados dois pontos $\left(x_{1}, y_{1}\right)$ e $\left(x_{2}, y_{2}\right)$ quaisquer, a reta que os contém (satisfaz).

Poderíamos avançar na dificuldade e considerar a seguinte situação II: seja um conjunto com $n$ pontos quaisquer, digamos $A=\left\{\left(x_{1}, y_{1}\right),\left(x_{2}, y_{2}\right), \cdots,\left(x_{n}, y_{n}\right)\right\}, n \in \mathbb{N}, n \geq 2$. Qual é a reta que contém os pontos do conjunto $A$ ? Esse segundo caso pode não ter solução, pelo simples fato de que dois pontos distintos determinam uma única reta (o que implica que o problema I possui solução única, no caso dos pontos serem distintos), então, para $n>2$, não há garantia de que todos os pontos estarão alinhados. Nesses termos, podemos considerar também a existência de uma reta que melhor se ajusta aos pontos do conjunto $A$, isto é, que melhor descreve, se enquadra ao conjunto de pontos dados.

Observe que no caso I, a função que se almeja deve satisfazer exatamente os pontos dados, a isso dá-se o nome de interpolação. No problema II, o que se espera é que a reta ajustada acompanhe o padrão dos dados (conjunto de pontos), neste caso, um padrão linear. É preciso dar significado a ideia de ajuste; esse é o objetivo da próxima seção.

\section{Desenvolvimento}

Para encontrar esta reta, com equação

$$
y=a x+b,
$$

é necessário definir o critério de otimização que dá significado ao termo "melhor ajuste". A reta definida pela equação (1) aproxima os dados e por isso existe um erro inerente à aproximação. $\mathrm{O}$ critério de otimização é que esse erro seja o menor possível, isto é, que seja mínimo. Então, basta definir uma função que meça o erro cometido na aproximação.

Cada ordenada $y_{i}$ dos pontos de $A$ será aproximada por $\hat{y}_{i}=a x_{i}+b$, de modo que o erro quadrático no $i$-ésimo ponto seja $\left(y_{i}-\hat{y}_{i}\right)^{2}, 1 \leq i \leq n$. Então, o erro total cometido na aproximação será

$$
E(a, b)=\sum_{i=1}^{n}\left(y_{i}-a x_{i}-b\right)^{2}
$$

ou, após seu desenvolvimento,

$$
E(a, b)=a^{2} \sum_{i=1}^{n} x_{i}^{2}+n b^{2}+2 a b \sum_{i=1}^{n} x_{i}-2 a \sum_{i=1}^{n} x_{i} y_{i}-2 b \sum_{i=1}^{n} y_{i}+\sum_{i=1}^{n} y_{i}^{2} .
$$

Desejamos que a soma dos quadrados dos desvios, isto é, a função dada pela equação (3) seja mínima. Em outras palavras, buscamos minimizar (determinar o mínimo) da função (3). Teria ela 
esse mínimo desejado? Com o objetivo de responder essa pergunta, necessitamos definir o conceito de mínimo (máximo) global em funções de duas variáveis como $E(a, b)$ :

Definição 2. Uma função $f$, com domínio em $\mathbb{R}^{2}$, possui um mínimo global quando se é possível encontrar um par $\left(x_{m}, y_{m}\right) \in \mathbb{R}^{2}$, tal que $f\left(x_{m}, y_{m}\right) \leq f(x, y)$, qualquer que seja $(x, y) \in \mathbb{R}^{2}$. Analogamente, o máximo global é definido a partir da possibilidade de se poder determinar $\left(x_{M}, y_{M}\right) \in \mathbb{R}^{2}$ de tal modo que $f\left(x_{M}, y_{M}\right) \geq f(x, y)$, para todo $(x, y) \in \mathbb{R}^{2}$.

\subsection{Determinando o Ponto de Mínimo}

Numa perspectiva intuitiva e exploratória, supondo a existência desse ponto de mínimo $\left(a_{m}, b_{m}\right)$, podemos fixar $b=b_{m}$ na função erro $E$, obtendo uma função quadrática na variável $a$, com coeficiente dominante positivo:

$$
E_{b_{m}}(a)=\left(\sum_{i=1}^{n} x_{i}^{2}\right) a^{2}+2\left(b_{m} \sum_{i=1}^{n} x_{i}-\sum_{i=1}^{n} x_{i} y_{i}\right) a+\left(n b_{m}^{2}+\sum_{i=1}^{n} y_{i}^{2}-2 b_{m} \sum_{i=1}^{n} y_{i}\right) .
$$

Analogamente, fixando $a=a_{m}$ em (3), obtemos a função também quadrática na variável $b$ de coeficiente dominante positivo:

$$
E_{a_{m}}(b)=n b^{2}+2\left(a_{m} \sum_{i=1}^{n} x_{i}-\sum_{i=1}^{n} y_{i}\right) b+\left(a_{m}^{2} \sum_{i=1}^{n} x_{i}^{2}+\sum_{i=1}^{n} y_{i}^{2}-2 a_{m} \sum_{i=1}^{n} x_{i} y_{i}\right) .
$$

Nestes termos, (4) e (5) possuem pontos de mínimo, conforme teoria das funções quadráticas que estabelece a existência de valor mínimo a partir do fato de o coeficiente dominante ser positivo. Seus pontos de mínimo são $a_{m}$ e $b_{m}$, respectivamente (prove isso), e são conhecidamente dados conforme equação:

$$
a_{m}=\frac{-2\left(b_{m} \sum_{i=1}^{n} x_{i}-\sum_{i=1}^{n} x_{i} y_{i}\right)}{2 \sum_{i=1}^{n} x_{i}^{2}} \quad \text { e } \quad b_{m}=\frac{-2\left(a_{m} \sum_{i=1}^{n} x_{i}-\sum_{i=1}^{n} y_{i}\right)}{2 n} .
$$

Assim, combinando as equações em (6) na forma de um sistema linear, chega-se aos coeficientes angular e linear da reta (1) desejada:

$$
a_{m}=\frac{n \sum_{i=1}^{n} x_{i} y_{i}-\sum_{j=1}^{n} y_{j} \sum_{i=1}^{n} x_{i}}{n \sum_{i=1}^{n} x_{i}^{2}-\left(\sum_{i=1}^{n} x_{i}\right)^{2}} \quad \text { e } \quad b_{m}=\frac{\sum_{j=1}^{n} y_{j} \sum_{i=1}^{n} x_{i}^{2}-\sum_{i=1}^{n} x_{i} y_{i} \sum_{j=1}^{n} x_{j}}{n \sum_{i=1}^{n} x_{i}^{2}-\left(\sum_{i=1}^{n} x_{i}\right)^{2}} .
$$

O desenvolvimento acima tem por hipótese a existência do ponto de mínimo. Dessa forma, se $E$ possui mínimo, então ele ocorre no ponto $(a, b)$ dado conforme equação (7). Agora qual é a condição para que $E$ possua um valor mínimo, isto é, quando que se pode afirmar a existência do valor mínimo? 


\section{Condição Suficiente para Existência de Ponto de Mínimo}

Considere nos desenvolvimentos a seguir o caso geral de (3), pela função polinomial de duas variáveis $f: \mathbb{R}^{2} \rightarrow \mathbb{R}$, tal que

$$
f(x, y)=a_{1} x^{2}+a_{2} y^{2}+a_{3} x y+a_{4} x+a_{5} y+a_{6}, \text { com } a_{1} \neq 0 \text { e } a_{2} \neq 0 .
$$

Estabeleceremos o seguinte

Teorema 1. i) O coeficiente $a_{1}$ é positivo (negativo), se e somente se, para todo $y_{0} \in \mathbb{R}$ fixo, existe um ponto $\left(x_{m}, y_{0}\right)$ tal que $f\left(x_{m}, y_{0}\right) \leq f\left(x, y_{0}\right)\left(f\left(x_{m}, y_{0}\right) \geq f\left(x, y_{0}\right)\right)$, qualquer que seja $x \in \mathbb{R}$;

ii) $\mathrm{O}$ coeficiente $\mathrm{a}_{2}$ é positivo (negativo), se e somente se, para todo $x_{0} \in \mathbb{R}$ fixo, existe um ponto $\left(x_{0}, y_{m}\right)$ tal que $f\left(x_{0}, y_{m}\right) \leq f\left(x_{0}, y\right)\left(f\left(x_{0}, y_{m}\right) \geq f\left(x_{0}, y\right)\right)$, qualquer que seja $y \in \mathbb{R}$.

Demonstração. i) Com efeito, fixando-se $y_{0}$ em $f$, obtemos uma função real $g$

$$
f\left(x, y_{0}\right)=a_{1} x^{2}+\left(a_{3} y_{0}+a_{4}\right) x+\left(a_{2} y_{0}^{2}+a_{5} y_{0}+a_{6}\right)=b_{1} x^{2}+b_{2} x+b_{3}=g(x),
$$

quadrática, com coeficiente dominante positivo e que, portanto, admite valor mínimo, isto é, existe

$$
x_{m}=-\frac{a_{3} y_{0}+a_{4}}{2 a_{1}},
$$

real tal que, conforme Definição 1,

$$
f\left(x_{m}, y_{0}\right)=g\left(x_{m}\right) \leq g(x)=f\left(x, y_{0}\right), \quad \text { qualquer que seja } x \text { real. }
$$

Por outro lado, se existe um ponto $\left(x_{m}, y_{0}\right)$ tal que $f\left(x_{m}, y_{0}\right) \leq f\left(x, y_{0}\right)$, qualquer que seja $x \in \mathbb{R}$, então

$$
\begin{aligned}
f\left(x_{m}, y_{0}\right)-f\left(x, y_{0}\right) & =a_{1} x_{m}^{2}+a_{3} x_{m} y_{0}+a_{4} x_{m}-a_{1} x^{2}-a_{3} x y_{0}-a_{4} x, \\
& =a_{1} x_{m}^{2}+\left(a_{3} y_{0}+a_{4}\right) x_{m}-a_{1} x^{2}-\left(a_{3} y_{0}+a_{4}\right) x, \\
& =-a_{1} x_{m}^{2}-a_{1} x^{2}+2 a_{1} x x_{m}, \\
& =-a_{1}\left(x_{m}-x\right)^{2} \leq 0, \quad \forall x \in \mathbb{R} .
\end{aligned}
$$

Assim, para que a inequação em (11) seja verdadeira é necessário e suficiente que $a_{1}>0$.

ii) A demonstração é análoga. Observamos apenas que $y_{m}$ é dado por

$$
y_{m}=-\frac{a_{3} x_{0}+a_{5}}{2 a_{2}} .
$$

Observação 1. A demonstração da implicação de ida $(\Rightarrow)$ é simples consequência de que uma função quadrática possui um mínimo (máximo) (concavidade para cima (baixo)) se, e somente se, o seu coeficiente dominante é positivo (negativo). Neste caso, o ponto de mínimo (máximo), que portanto é único, fica completamente determinado pelos coeficientes da função quadrática, conforme equações (9) e (12). 
Observação 2. A implicação de volta $(\Leftarrow)$ pode ser menos restritiva, conforme sua demonstração, de modo que as hipóteses "para todo $y_{0} \in \mathbb{R}$..."e "para todo $x_{0} \in \mathbb{R}$..."podem ser substituídas por "existe $y_{0} \in \mathbb{R}$..."e "existe $x_{0} \in \mathbb{R}$...", respectivamente. Tais implicações tornam-se então:

i) Se existe $y_{0} \in \mathbb{R}$ tal que, para algum ponto $\left(x_{m}, y_{0}\right)$, tem-se $f\left(x_{m}, y_{0}\right) \leq f\left(x, y_{0}\right)\left(f\left(x_{m}, y_{0}\right) \geq\right.$ $\left.f\left(x, y_{0}\right)\right)$, qualquer que seja $x \in \mathbb{R}$, então o coeficiente $a_{1}$ é positivo (negativo).

ii) Se existe $x_{0} \in \mathbb{R}$ tal que, para algum ponto $\left(x_{0}, y_{m}\right)$, tem-se $f\left(x_{0}, y_{m}\right) \leq f\left(x_{0}, y\right)\left(f\left(x_{0}, y_{m}\right) \geq\right.$ $\left.f\left(x_{0}, y\right)\right)$, qualquer que seja $y \in \mathbb{R}$, então o coeficiente $a_{2}$ é positivo (negativo).

Observação 3. Importante notar também que $x_{m}$ e $y_{m}$ obtidos nos itens i) e ii), respectivamente, não são arbitrários e dependem, cada qual, explicitamente dos pontos $y_{0}$ e $x_{0}$, conforme equações (9) e (12), respectivamente.

Corolário 1. Se a função f admite um mínimo (máximo) global, então $a_{1}>0$ e $a_{2}>0\left(a_{1}<0\right.$ e $\left.a_{2}<0\right)$. Além disso, $\left(x_{m}, y_{m}\right)$ dado no Teorema 1 é o ponto de mínimo (máximo).

Demonstração. Com efeito, se $f$ possui mínimo então existe $\left(x_{0}, y_{0}\right)$ em $\mathbb{R}^{2}$ tal que

$$
f\left(x_{0}, y_{0}\right) \leq f(x, y), \quad \text { qualquer que seja }(x, y) \in \mathbb{R}^{2} .
$$

De modo particular, podemos obter

$$
f\left(x_{0}, y_{0}\right) \leq f\left(x_{0}, y\right), \quad \forall y \in \mathbb{R} \quad \text { e } \quad f\left(x_{0}, y_{0}\right) \leq f\left(x, y_{0}\right), \quad \forall x \in \mathbb{R} .
$$

Assim, pelo Teorema 1, concluímos que $a_{1}$ e $a_{2}$ devem ser positivos.

O fato de o ponto de mínimo ter coordenadas

$$
x_{m}=-\frac{a_{3} y_{m}+a_{4}}{2 a_{1}} \quad \text { e } \quad y_{m}=-\frac{a_{3} x_{m}+a_{5}}{2 a_{2}}
$$

ocorre pelas desigualdades em (14), das equações (9) e (12) e ainda da unicidade de $x_{m}$ e $y_{m}$.

Para avançar na compreensão de $f$, analisaremos a situação em que $a_{3}=0$ e inexiste termo misto. Para isso, demonstraremos o seguinte

Teorema 2. Se $a_{1}>0\left(a_{1}<0\right), a_{2}>0\left(a_{2}<0\right)$ e $a_{3}=0$, então $f$ possuí mínimo (máximo) global.

Demonstração. A condição $a_{3}=0$ implica que os pontos $x_{m}$ e $y_{m}$ dados pelo Teorema 1 não dependem de $x_{0}$ e $y_{0}$, respectivamente, conforme equações (9) e (12):

$$
x_{m}=-\frac{a_{4}}{2 a_{1}} \quad \text { e } \quad y_{m}=-\frac{a_{5}}{2 a_{2}} .
$$

Dessa forma, $f$ avaliada em $\left(x_{m}, y_{m}\right)$ dados por $(16)$ e com a hipótese de que $a_{1}>0$ e $a_{2}>0$, resulta em

$$
\begin{aligned}
f\left(x_{m}, y_{m}\right) & =-a_{1}\left(\frac{a_{4}}{2 a_{1}}\right)^{2}-a_{2}\left(\frac{a_{5}}{2 a_{2}}\right)^{2}+a_{6} \\
& \leq-a_{1}\left(\frac{a_{4}}{2 a_{1}}\right)^{2}-a_{2}\left(\frac{a_{5}}{2 a_{2}}\right)^{2}+a_{6}+a_{1}\left(x+\frac{a_{4}}{2 a_{1}}\right)^{2}+a_{2}\left(y+\frac{a_{5}}{2 a_{2}}\right)^{2}, \\
& =f(x, y), \quad \forall(x, y) \in \mathbb{R}^{2} .
\end{aligned}
$$


Concluímos, nos termos da Definição 2, que $f$ possui ponto de mínimo global que é dado por $\left(x_{m}, y_{m}\right)$, conforme equações $(16)$.

O caso da existência de máximo global em que $a_{1}<0$ e $a_{2}<0$ prova-se de maneira análoga.

Vejamos o caso geral no seguinte

Teorema 3. Se $a_{1}>0\left(a_{1}<0\right), a_{2}>0\left(a_{2}<0\right)$ e $a_{1} a_{2}-\left(a_{3} / 2\right)^{2}>0$, então $f$ possui mínimo (máximo) global em $\left(x_{m}, y_{m}\right)$.

Demonstração. Com efeito:

$$
\begin{aligned}
f(x, y)= & \frac{a_{1}}{2}\left[\left(x+\frac{a_{3} y+a_{4}}{2 a_{1}}\right)^{2}-\left(\frac{a_{3} y+a_{4}}{2 a_{1}}\right)^{2}\right]+\frac{a_{2}}{2}\left[\left(y+\frac{a_{3} x+a_{5}}{2 a_{2}}\right)^{2}-\left(\frac{a_{3} x+a_{5}}{2 a_{2}}\right)^{2}\right] \\
& +\frac{a_{1}}{2} x^{2}+\frac{a_{4}}{2} x+\frac{a_{2}}{2} y^{2}+\frac{a_{5}}{2} y+a_{6}, \\
\geq & -\frac{a_{1}}{2}\left(\frac{a_{3} y+a_{4}}{2 a_{1}}\right)^{2}-\frac{a_{2}}{2}\left(\frac{a_{3} x+a_{5}}{2 a_{2}}\right)^{2}+\frac{a_{1}}{2} x^{2}+\frac{a_{4}}{2} x+\frac{a_{2}}{2} y^{2}+\frac{a_{5}}{2} y+a_{6}, \\
= & \left(\frac{a_{1} a_{2}-\left(a_{3} / 2\right)^{2}}{2 a_{2}}\right) x^{2}+\left(\frac{a_{1} a_{2}-\left(a_{3} / 2\right)^{2}}{2 a_{1}}\right) y^{2}+\left(\frac{2 a_{2} a_{4}-a_{3} a_{5}}{4 a_{2}}\right) x \\
& +\left(\frac{2 a_{1} a_{5}-a_{3} a_{4}}{4 a_{1}}\right) y+\left(a_{6}-\frac{a_{4}^{2}}{8 a_{1}}-\frac{a_{5}^{2}}{8 a_{2}}\right) \\
= & g(x, y), \quad \forall(x, y) \in \mathbb{R}^{2} .
\end{aligned}
$$

Pelo Teorema 2, a função $g$ possui mínimo, logo, existe $\left(x_{0}, y_{0}\right)$ tal que

$$
g\left(x_{0}, y_{0}\right) \leq g(x, y) \leq f(x, y), \quad \forall(x, y) \in \mathbb{R}^{2},
$$

ou seja, a função $f$ é limitada inferiormente. Pelo Corolário 1, o mínimo de $g$ ocorre conforme equação (15), isto é,

$$
x_{0}=\frac{\left(-\frac{2 a_{2} a_{4}-a_{3} a_{5}}{4 a_{2}}\right)}{2\left(\frac{a_{1} a_{2}-\left(a_{3} / 2\right)^{2}}{2 a_{2}}\right)}=\frac{a_{3} a_{5}-2 a_{2} a_{4}}{4 a_{1} a_{2}-a_{3}^{2}} \quad \text { e } \quad y_{0}=\frac{-\left(\frac{2 a_{1} a_{5}-a_{3} a_{4}}{4 a_{1}}\right)}{2\left(\frac{a_{1} a_{2}-\left(a_{3} / 2\right)^{2}}{2 a_{1}}\right)}=\frac{a_{3} a_{4}-2 a_{1} a_{5}}{4 a_{1} a_{2}-a_{3}^{2}} .
$$

O par $\left(x_{0}, y_{0}\right)$ dado pela equação (20) corresponde à solução do sistema linear constituído pelas equações em (15):

$$
\left[\begin{array}{rr}
2 a_{1} & a_{3} \\
a_{3} & 2 a_{2}
\end{array}\right]\left[\begin{array}{l}
x_{m} \\
y_{m}
\end{array}\right]=\left[\begin{array}{l}
-a_{4} \\
-a_{5}
\end{array}\right]
$$

Este sistema possui solução única, conforme hipótese do teorema que garante que o determinante da matriz de coeficientes é diferente de zero. Assim, temos que $\left(x_{0}, y_{0}\right)=\left(x_{m}, y_{m}\right)$. Por outro lado, conforme equação (18),

$$
f(x, y)=g(x, y)+\frac{a_{1}}{2}\left(x+\frac{a_{3} y+a_{4}}{2 a_{1}}\right)^{2}+\frac{a_{2}}{2}\left(y+\frac{a_{3} x+a_{5}}{2 a_{2}}\right)^{2}
$$


e por isso, segue-se que

$$
f\left(x_{m}, y_{m}\right)=g\left(x_{m}, y_{m}\right)
$$

ao passo que da inequação em (19), concluímos que

$$
f\left(x_{m}, y_{m}\right) \leq f(x, y), \quad \forall(x, y) \in \mathbb{R}^{2} .
$$

A função $E(a, b)$ satisfaz as condições dadas pelo Teorema 3 se $n \geq 2$ e se pelo menos um dos $x_{i}$ for diferente dos demais $x_{j}$, pois nesse caso conseguimos garantir que ambos $a_{1}$ e $a_{2}$ são positivos:

$$
a_{1}=\sum_{i=1}^{n} x_{i}^{2}>0 \quad \text { e } \quad a_{2}=n>0
$$

A desigualdade $a_{1} a_{2}-\left(a_{3} / 2\right)^{2}>0$ pode ser verificada por indução. Com efeito, provaremos inicialmente a seguinte igualdade:

$$
a_{1} a_{2}-\left(a_{3} / 2\right)^{2}=n \sum_{i=1}^{n} x_{i}^{2}-\left(\sum_{i=1}^{n} x_{i}\right)^{2}=\sum_{i=1, j \neq i}^{n}\left(x_{i}-x_{j}\right)^{2} .
$$

Para $n=2$ temos que

$$
a_{1} a_{2}-\left(a_{3} / 2\right)^{2}=2\left(x_{1}^{2}+x_{2}^{2}\right)-\left(x_{1}+x_{2}\right)^{2}=\left(x_{1}-x_{2}\right)^{2} .
$$

Agora, supondo verdadeira a seguinte hipótese de indução

$$
n \sum_{i=1}^{n} x_{i}^{2}-\left(\sum_{i=1}^{n} x_{i}\right)^{2}=\sum_{i=1, j \neq i}^{n}\left(x_{i}-x_{j}\right)^{2},
$$

então,

$$
\begin{aligned}
(n+1) \sum_{i=1}^{n+1} x_{i}^{2}-\left(\sum_{i=1}^{n+1} x_{i}\right)^{2} & =(n+1) \sum_{i=1}^{n} x_{i}^{2}+(n+1) x_{n+1}^{2}-\left(x_{n+1}+\sum_{i=1}^{n} x_{i}\right)^{2}, \\
& =\sum_{i=1, j \neq i}^{n}\left(x_{i}-x_{j}\right)^{2}+n x_{n+1}^{2}+\sum_{i=1}^{n} x_{i}^{2}-2 x_{n+1} \sum_{i=1}^{n} x_{i}, \\
& =\sum_{i=1, j \neq i}^{n}\left(x_{i}-x_{j}\right)^{2}+\sum_{i=1}^{n}\left(x_{n+1}-x_{i}\right)^{2}, \\
& =\sum_{i=1, j \neq i}^{n+1}\left(x_{i}-x_{j}\right)^{2} .
\end{aligned}
$$

ou seja,

$$
a_{1} a_{2}-\left(a_{3} / 2\right)^{2}=\sum_{i=1, j \neq i}^{n}\left(x_{i}-x_{j}\right)^{2}, \quad n \geq 2,
$$

e como por hipótese pelo menos um $x_{i}$ é diferente de algum dos demais $x_{j}$, temos que $a_{1} a_{2}-$ $\left(a_{3} / 2\right)^{2}>0$. Concluímos, com isso, a prova do seguinte 
Teorema 4. Considere o conjunto $A=\left\{\left(x_{1}, y_{1}\right),\left(x_{2}, y_{2}\right), \cdots,\left(x_{n}, y_{n}\right)\right\}$, dado de tal forma que exista um $x_{i} \neq x_{j}$, em que $i, j \in\{1, \cdots, n\}$, e com $2 \leq n \in \mathbb{N}$. A função $E(a, b)$, definida pela equação (3) sobre os pontos do conjunto A, possui mínimo global.

Observação 4. Observamos que para $n=1$, a condição $a_{1} a_{2}-\left(a_{3} / 2\right)^{2}>0$ não é satisfeita, visto que nesse caso teríamos $a_{1} a_{2}-\left(a_{3} / 2\right)^{2}=x_{1}^{2}-x_{1}^{2}=0$, conforme equação (25).

Observação 5. É possível concluirmos que $a_{1} a_{2}-\left(a_{3} / 2\right)^{2} \geq 0$ pela desigualdade de Cauchy-Schwarz (LIMA, 2008, p20), conforme equação (25).

\section{Considerações Finais}

Desenvolvemos o método de mínimos quadrados lineares utilizando os temas abordados no ensino médio: funções quadráticas e desigualdades. A presente estratégia constitui uma aplicação valiosa que pode ser inserida no contexto de problemas de otimização em nível de ensino médio, sobretudo quando desenvolvida na forma de projetos de ensino e iniciação científica em classes de cursos técnicos de tecnologia integrados ao ensino médio.

Em termos práticos, tendo em vista o contexto do ensino médio, poder-se-ia abordar apenas a determinação do ponto de mínimo a partir da hipótese de existência desse mínimo (primeira subseção junto à seção Desenvolvimento), visto que a construção da condição suficiente para existência de mínimo (segunda subseção da mesma seção) possui maiores detalhes em sua teoria.

A construção de um pseudocódigo que computa os parâmetros do modelo, coeficientes $a$ e $b$ da reta de equação (1), constitui uma primeira aplicação que se poderia considerar, sobretudo em cursos técnicos que adotam alguma linguagem de programação em seus currículos. Um segundo tema a ser considerado é aquele que se preocupa com o crescimento populacional. Para a aplicação, é possível obter os dados relativos ao censo demográfico brasileiro através de consulta junto à página do Ibge [4]. Com as devidas adaptações ${ }^{1}$, um modelo exponencial $y=a \mathrm{e}^{b x}$ pode ser analogamente desenvolvido a fim de se obter uma função útil para se realizar previsões da população brasileira. Um terceiro tema que pode ser abordado, da tecnologia de hardware, é aquele associado ao conceito de latência ${ }^{2}$ CAS (Column Address Strobe). As variáveis latência CAS e velocidade possuem um padrão linear que pode ser estudado via mínimos quadrados (os dados necessários para o estudo, bem como maiores informações, estão disponíveis em [3]).

\section{Agradecimentos}

Agradecimentos ao professor Ricardo David de Morais da Silva pelas discussões e contribuições, bem como aos estudantes Matheus Eduardo dos Santos e Keslene Lima que trabalharam com o tema em suas iniciações científicas.

\footnotetext{
${ }^{1} \mathrm{Na}$ equação (2), no lugar de se obter $E(a, b)$ calculando-se o erro entre $y$ e $\hat{y}$, obtenha-o calculando o logaritmo natural $\ln y$ e $\ln \hat{y}$ dos mesmos. Isso tornará o desenvolvimento idêntico ao presente. Para mais detalhes consultar Burden e Faires (2008, p465).

${ }^{2}$ Refere-se ao intervalo de tempo, medido pelo número de ciclos de clock, em que um comando é inserido e executado [3].
} 


\section{Referências}

[1] Burden, R. L.; Faires, J. D. Análise Numérica. São Paulo: Cengage Learning, 2008.

[2] Chavante, E.; Prestes, D. Quadrante Matemática, $1^{o}$ Ano: Ensino Médio. São Paulo: Edições SM, 2016.

[3] MICRON TECHNOLOGY. Speed vs. Latency: Why CAS latency isn't an accurate measure of memory performance. Disponível em: https://www.crucial.com/usa/en/memory-performancespeed-latency. Acesso em: 05 setembro 2018.

[4] Ibge- Instituto Brasileiro de Geografia e Estatística. Disponível em: https://www.ibge.gov.br. Acesso em: 31 maio 2019.

[5] Iezzi, G., Murakami, C. Fundamentos de Matemática Elementar - Conjuntos, Funções. São Paulo: Atual Ed., 1977.

[6] Iezzi, G.; Dolce, O.; Degenszajn, D.; Périgo, R.; Almeida, N. Matemática: Ciência e Aplicações, Volume 1: Ensino Médio. São Paulo: Saraiva, 2013.

[7] Lima, E. L. Análise Real Volume 1. Funções de Uma Variável Real. Rio de Janeiro: IMPA, 2008.

[8] Paiva, M. Matemática: Paiva. São Paulo: Moderna, 2015.

[9] Souza, J. R.; Garcia, J. S. R. \# Contato Matemática, $1^{o}$ Ano. São Paulo: FTD, 2016.

Adriano R. de Melo Instituto Federal de Educação, Ciência e Tecnologia Catarinense Araquari/SC <adriano.melo@ifc.edu.br>

Recebido: 23/04/2019 Publicado: 02/07/2019 